Published in final edited form as:

Nat Photonics. 2020 July ; 14(7): 439-445. doi:10.1038/s41566-020-0608-y.

\title{
Transscleral Optical Phase Imaging of the Human Retina
}

\author{
T. Laforest ${ }^{\# 1,6}$, M. Künzi ${ }^{\# 1,6}$, L. Kowalczuk ${ }^{2,3}$, D. Carpentras ${ }^{1}$, F. Behar-Cohen ${ }^{2,4,5}$, C. \\ Moser ${ }^{1, *}$
}

${ }^{1}$ Laboratory of Applied Photonic Devices (LAPD), School of Engineering, École Polytechnique Fédérale de Lausanne (EPFL), Lausanne, Switzerland ${ }^{2}$ Faculty of Biology and Medicine, University of Lausanne, Lausanne, Switzerland ${ }^{3} J u l e s-G o n i n$ Eye Hospital, Fondation Asile des aveugles, Lausanne, Switzerland ${ }^{4}$ Centre de Recherche des Cordeliers, Inserm, USPC, Université de Paris, Sorbonne Université, From physiopathology of ocular diseases to clinical developments, F-75006 Paris, France ${ }^{5}$ Ophtalmopole, Hôpital Cochin, Assistance Publique Hôpitaux de Paris, Université de Paris, Paris, France ${ }^{6}$ EarlySight SA, EPFL Innovation Park, Lausanne, Switzerland

\# These authors contributed equally to this work.

\section{Abstract}

In-vivo observation of the human retina at the cellular level is crucial to detect the first signs of retinal diseases and properly treat them. Despite the phenomenal advances in adaptive optics (AO) systems, clinical imaging of many retinal cells is still elusive due to the low signal-to-noise ratio induced by transpupillary illumination.

We present a transscleral optical phase imaging (TOPI) method, which relies on high-angle oblique illumination of the retina, combined with $\mathrm{AO}$, to enhance cell contrast. Examination of eleven healthy volunteer eyes, without pupil dilation, shows the ability of this method to produce in-vivo images of retinal cells, from the retinal pigment epithelium to the nerve fibre layer. This method also allows the generation of high-resolution label-free ex-vivo phase images of flat-

Users may view, print, copy, and download text and data-mine the content in such documents, for the purposes of academic research, subject always to the full Conditions of use:http://www.nature.com/authors/editorial_policies/license.html\#terms

*Materials and Correspondence: Correspondence and requests for materials should be addressed to christophe.moser@epfl.ch.

Code availability

The code for performing the reconstruction process may be requested from the authors.

Author contributions

TL designed and built the ex-vivo microscope, obtained the ex-vivo results, built the in-vivo device, wrote the code for ex-vivo and invivo processing, and wrote the paper. MK designed and built the in-vivo device, wrote the code for in-vivo processing, and wrote the paper. DC developed the theoretical model and wrote the paper. LK provided and prepared the ex-vivo samples, participated in the interpretation to the phase images, and wrote the paper. FBC supervised the project and wrote the paper. CM designed the experiment, supervised the project, and wrote the paper.

Competing interests

The authors TL, MK, FBC and CM are involved in a company (EarlySight SA, Switzerland) aiming to commercialize the technology. A patent application has been submitted with number WO2017195163A1.

Data and materials availability

All data needed to evaluate the conclusions in this paper are present in the paper and/or the Supplementary Materials. The data that support the plots within this paper and other findings of this study are available from the corresponding author upon reasonable request. 
mounted retinas. The $4.4^{\circ} \times 4.4^{\circ}$ field-of-view in-vivo images are recorded in less than 10 seconds, opening new avenues in the exploration of healthy and diseased retinas.

\section{Introduction}

Retinal diseases are the major cause of blindness in industrialized countries. For example, an estimated total of 196 million people will be affected by age related macular degeneration in $2020^{1}$. While tremendous effort is being made to develop novel therapeutic strategies to rescue retinal neurons and the Retinal Pigment Epithelium (RPE), optimal means for evaluating the effects of such treatments are still missing. The instruments used in eye clinics for routine eye fundus examination are not able to observe the minute changes in cell morphology that are present during early stages of the disease degenerative process.

The retina is an intricate and complex tissue composed of many layers. Individual cell imaging is very challenging due to many constraints such as ocular aberration reducing lateral resolution, eye motion artefacts and the lack of contrast of transparent cells. Another limitation is that most of the light entering the pupil is either absorbed or reflected at the photoreceptor segments interface ${ }^{2,3}$, overwhelming the weak signal back-scattered from the neuronal or RPE cells. The photoreceptor signal is maximum for an illumination beam entering the eye pupil centre and decreases sharply when entering at its edge. This angulardependent reflection of the retina is commonly referred as optical Stiles-Crawford effect $(\mathrm{SCE})^{3}$.

The light reflected by the photoreceptors renders their observation possible using adaptive optics (AO) to correct ocular aberrations combined with flood illumination ${ }^{4-8}$ optical coherence tomography (OCT) ${ }^{9}$ or scanning laser ophthalmoscopy (SLO ${ }^{10}$. In recent works, optical methods to observe the neuroretina and the RPE cells were proposed using AO-SLO with offset aperture ${ }^{11-14}$, split detector ${ }^{15,16}$, darkfield ${ }^{17}$, autofluorescence ${ }^{18}$, or AO$\mathrm{OCT}^{9,19}$. Despite the high-quality results obtained, these methods suffer either from a very small field-of-view (FOV), a lack of image clarity, safety concerns or an acquisition time too long for a clinical use.

Here, we propose a modality, called transscleral optical phase imaging (TOPI) that provides cellular-resolution label-free high-contrast images of the retinal layers over a large FOV without the drawback of a long exposure time. This method is based on transscleral flood illumination of the retina, which greatly increases the signal to noise ratio (SNR) of many retina structures as compared to trans-pupillary illumination. The light transmitted through the sclera creates an oblique illumination of the posterior retina, that is then imaged using a trans-pupillary AO full-field camera system. We demonstrate in healthy volunteers without pupil dilation that TOPI provides images of RPE cells and other retinal structures and enables cell quantification. We also demonstrate the potential of this technology on ex vivo samples using an experimental phase microscope with parameters similar to those of the in vivo apparatus. We show that label-free phase images of the cells in thick retina samples are matching the quality of confocal fluorescence microscopy. 


\section{Results}

\section{Transscleral illumination concept}

Transscleral illumination of the fundus produces an oblique beam that reaches the posterior layers of the eye (Fig. 1). The sclera transmits about $30 \%$ of the incident light when using near-infrared (NIR) light ${ }^{20}$, and the absorption and reflectance of the RPE-choroid complex vary with its pigmentation ${ }^{21,22}$. While crossing the sclera, the illumination light is scattered and diffused, producing a uniform highly divergent beam that reaches obliquely the posterior retina and illuminates it evenly.

Transscleral illumination presents two interesting properties that increase the low contrast of the retina observed with trans-pupil flood illumination. Firstly, due to the SCE, almost no high angle transscleral illumination light is coupled into the photoreceptors, allowing a large fraction of the light to reach the RPE layer. Secondly, no direct backscattered illumination light is collected by the imaging system because there is no overlap with the collection path located through the pupil. Thus, only the light multiply scattered by the different retina layers enters the optical system and reaches the camera, providing a darkfield imaging condition. The illumination angle is much larger than what is obtainable via illumination through the pupil, creating a non-uniform excitation of the retina spatial frequencies which enhances the contrast of transparent objects ${ }^{23,24}$.

\section{Description of the TOPI camera}

The optical diagram of the TOPI camera is shown in Fig. 2. The system combines transscleral illumination of the retina with a flood illumination AO fundus camera ${ }^{25}$. Two NIR (wavelength, $\lambda=810 \mathrm{~nm}$ ) light emitting diodes (LED), located on the nasal and temporal side of the eye, are imaged on the sclera surface and produce the transscleral illumination (Fig. 2b).

The rest of the system is composed of an AO closed-loop for ocular aberration correction, a camera (2D array detector, complementary metal oxide semiconductor (CMOS)) conjugated to the pupil for alignment purpose, a fixation target for retina location stabilization, and a scientific grade camera (sCMOS) for recording the high-resolution retina images. The details of the components used in the system are provided in the Materials and Methods section.

\section{In vivo imaging of the RPE}

The RPE layer image from a healthy volunteer taken with TOPI is exemplified in Fig. 3a (see Supplementary Fig. 1 for images in two additional volunteers). Due to the low SNR of the raw images coming from the retina, the in-vivo TOPI image was processed using a recorded sequence of 100 images taken within a total acquisition time of 9 seconds. The processing procedure of the sequence leading to a final image is described in the Materials and Methods section. Individual RPE cells are clearly visible on the full image with a dark centre and bright edges due to the absorbing pigments in the cell soma. The low reflectivity of the cone mosaic caused by the high angle illumination is not shadowing the signal from the RPE cells underneath. Due to the large depth-of-field $(\sim 30 \mu \mathrm{m})$, a lower spatial 
frequency bright-and-dark "cloudy" modulation is partially overlapping the RPE cell mosaic. This unwanted signal is coming from the non-uniform pigmentation of the epithelium and from the out-of-focus light scattered from the choroid ${ }^{17}$. This modulation can be partially removed by post-processing the final image with a high-pass filter, as illustrated in the magnified inset (Fig. 3b).

Fig. 3a illustrates the wide field-of-view image $\left(4.4^{\circ} \times 4.4^{\circ}\right)$ obtained with the TOPI system in less than 10 seconds while providing cellular resolution. In comparison, an AO-OCT system of similar spatial resolution has a significantly smaller field of view of $1.5^{\circ} \times 1.5^{\circ}$ and takes several minutes to obtain a similar image ${ }^{19}$.

The system allows imaging over a range of $\pm 15^{\circ}$ both vertically and horizontally (Supplementary Fig. 2). The SNR analysis performed on a sequence of raw images shows that an acquisition time as low as two seconds provides a suitable TOPI image for RPE quantification (Supplementary Fig. 3).

\section{RPE cells size and density}

RPE imaging was performed in eleven healthy volunteers (from 19 to 49 years-old). No major difference was noticed in the RPE images from the subjects with different skin pigmentations, demonstrating that TOPI could be used in patients of different phototypes ${ }^{26}$. The RPE cells quantification between $2^{\circ}$ and $12^{\circ}$ eccentricity gives an average area of $241 \pm$ $29 \mu^{2}$ (Supplementary Fig. 4A) for an average density of $4371 \pm 460$ cells $/ \mathrm{mm}^{2}$

(Supplementary Fig. 4B). Interestingly, the youngest participant presented the highest density of RPE cells, whereas the oldest participant presented the lowest density (Supplementary Fig. 4C).

This quantitative analysis matched the values found in the literature at these eccentricities ${ }^{9,19}$. One in-vivo autofluorescence AO-SLO study provided a RPE cell density of 5890 cells $/ \mathrm{mm}^{2}\left(168 \mu^{2}\right.$ area) and 5630 cells $/ \mathrm{mm}^{2}\left(195 \mu \mathrm{m}^{2}\right.$ area $)$ at $5^{\circ}$ and $6.25^{\circ}$ respectively ( 2 subjects $)^{27}$. Another AO-OCT study reported a mean density at $3^{\circ}$ of $4975 \pm$ 651 cells $/ \mathrm{mm}^{2}$ (6 subjects) ${ }^{19}$. Scoles et al. reported, from histological study in the macula, an average density of 5662 cells $/ \mathrm{mm}^{2}$ and an average cell area of $160 \mu \mathrm{m}^{2}$ for age below 60 (6 samples) ${ }^{28}$. These in-vivo and ex-vivo densities represent no significant difference with our data (probability value, $P>0.05$ ). Moreover, the row to row spacing of $13.8 \mu \mathrm{m}$ extracted from Fourier analysis of the images (Supplementary Fig. 5) is the same as a previously reported value obtained with $\mathrm{AO}-\mathrm{OCT}^{19}$.

\section{In-vivo imaging of the nerve fibre layer (NFL)}

By axially shifting the focal plane of the imaging system, other layers of the neuroretina can be observed (Fig. 4b-f). The location of each image is shown on the SLO fundus view (Fig. 4a). Images of the vascular layer (Fig. 4b,c) show that retinal capillaries as small as fewmicrons can be seen. The NFL is imaged at different eccentricities (Fig. 4d-f) where large axon bundles of 30-50 $\mu \mathrm{m}$ diameter are clearly visible and well-aligned in the direction of the optic nerve. The ability to observe very small capillaries and axons bundles comes from the dark-field effect of the transscleral illumination where the signal of the translucent structure is not buried under the illumination light. 


\section{Comparison between transscleral and transpupillary illumination}

The influence of the transscleral illumination on cell contrast is better understood by direct comparison with convention transpupillary illumination. A commercial AO retinal camera (model $\mathrm{rtx} 1^{\mathrm{TM}}$, Imagine Eyes), using flood transpupillary illumination was compared with the TOPI camera, using transscleral oblique illumination. Three layers located at different depths were imaged using both instruments, over the same area of the subject S10's fundus. Inner retina images (Fig. 5a,d) show the darkfield effect of the transscleral illumination with a bright vessel on a darker background. The transpupillary illumination in $\mathrm{rtx} 1^{\mathrm{TM}}$ allows better visualization of the photoreceptors than TOPI images (Fig. 5b,e). This is due to the optical SCE at the inner-outer segment interface which provides a high reflectivity when light is incident perpendicular to the retina ${ }^{6}$. However, the strong cone signal produced by transpupillary illumination masks the adjacent layers, particularly the posterior RPE where only defocused photoreceptor light is visible (Fig. 5c). On the contrary, with transscleral illumination, cones produce a very weak signal, and thus the RPE cells appear wellcontrasted (Fig. 5f). These results clearly confirm the very interesting properties of transscleral illumination for imaging the deepest layer of the retina.

\section{Oblique illumination for optical phase imaging microscopy}

To validate the potential of oblique illumination to observe retina tissues, we developed a custom microscope with parameters similar to those of in-vivo imaging (Supplementary Note 1, Supplementary Fig. 6). Images of flat-mounted retinas obtained with this optical phase imaging (OPI) microscope are free of in vivo problems such as eye movements and aberrations. Using multiple illumination points, quantitative phase information was extracted from the ex vivo samples (for details see the Materials and Methods section). Through a comparative study with other common microscopy techniques, we demonstrated the quantitative property and the high contrast of phase images, as well as the potential of this modality to observe the retinal cellular structures (Supplementary Note 2, Supplementary Fig. 7). In addition, cell counting was possible on all phase images of the layers of a $200 \mu \mathrm{m}-$ thick pig retina (Supplementary Note 3, Supplementary Fig. 8).

The OPI microscope was also equipped with high NA ( 0.4 or 0.65$)$ objectives to perform high resolution phase imaging. We observed rat retinal capillaries and their surrounding cells, in a correlative study between phase microscopy and fluorescence confocal microscopy, using vessels as landmarks. After specific labelling of the pericytes in a healthy retina, the comparison of both modalities demonstrates that OPI modality allows for the detection of pericytes, appearing as bright cells around retinal capillaries (Fig. 6a) exactly at the same location of the pericytes labelled in green in the fluorescence images (Fig. 6b-c). The nuclei counter-staining (Fig. 6c, in blue) shows that the proposed phase imaging method also allows for high contrast imaging of the nuclei. After specific labelling of microglial cells in a diseased retina presenting a dystrophy associated with telangiectasias ${ }^{29}$, the image correlation analysis between phase (Fig. 6d) and confocal (Fig. 6e, green) microscopy unequivocally demonstrates that high resolution phase imaging allows for the detection of these small cells as well. 


\section{Discussion}

Currently, the standard of care for retinal imaging is based on OCT and SLO. The first commercially available device integrating $\mathrm{AO}$ was based on flood illumination ( $\mathrm{rtx} 1^{\mathrm{TM}}$, Imagine Eyes). We describe herein a novel microscopy concept and instrument for fast invivo imaging of the retinal pigment epithelium with high contrast and cellular resolution.

To our knowledge, no other imaging system allows for the visualization of RPE cells within a few seconds. We see no technological barriers to create an easy-to-use clinical instrument because transscleral illumination requires the same accuracy in terms of beam alignment than transpupillary systems. On the contrary, for cellular-resolution imaging, AO-SLO and AO-OCT would be more difficult to implement in clinical setting. As presented in Supplementary Table 1, their FOV of $2^{\circ} \times 2^{\circ}$ is close to five times smaller than the current TOPI system FOV. Five times more images would be required to cover the same clinically relevant FOV. Moreover, the use of a non-scanning system makes the acquisition process more stable under the effects of eye motion, especially because AO-SLO and AO-OCT require measurement time of several minutes. For the latter, eye-motion artefacts become a problem, which is much less the case for a flood-illumination system, in which all the pixels composing the image are recorded simultaneously with a few-milliseconds acquisition time.

The speed of an eye examination is crucial for patient's comfort and becomes even more important when fixation capacity is reduced by retinal disease. The typical scan time for commercial clinical devices is a few seconds (2-3 s for Canon OCT-HS100, $2 \mathrm{~s}$ for Imagine Eyes rtx $1^{\mathrm{TM}}, 3 \mathrm{~s}$ for Optovue AngioVue, 19-38 ms for B-Scan for Heidelberg Spectralis OCT, and $2 \mathrm{~s}$ for Zeiss Cirrus HD-OCT). With less than ten seconds required for TOPI acquisition, the obtained contrast was sufficient to perform quantitative analyses on RPE cells. The full field flood imaging technique combined with transscleral illumination establishes an imaging modality that is several orders of magnitude faster than the AO-OCT technique when applied to weakly reflecting cells ${ }^{9,19}$. This difference is essentially due to the fact that TOPI uses the scattered light from the back of the eye, which is much more reflective than the transparent cells.

The light radiant exposure at the imaged area of the retina is comparable with existing technologies. For TOPI imaging, a single raw image requires an estimated radiant energy of $79 \mu \mathrm{J} / \mathrm{cm}^{2}$ at the retina, while the 100-images sequence needed to obtain the final image yields $7.9 \mathrm{~mJ} / \mathrm{cm}^{2}$. These values are similar to those of the commercial flood illumination device $\mathrm{rtx} 1^{\mathrm{TM}} 6$ and are lower than those used in a scanning system based on SLO or OCT (comparison with other AO imaging methods in Supplementary Fig. 9). The projected spot on the sclera has a power density that satisfies the safety standard for skin and ocular tissues (see the Materials and Methods section). We note that, in all previous studies related to highresolution imaging of the retina, averaging of raw data is also necessary to obtain high SNR.

In the present study, in-vivo examinations of eleven healthy volunteers with our TOPI set up demonstrated the feasibility of fast imaging of RPE cells but also the possibility of quantifying them accurately. As only one fundus area was observed in most subjects, more studies will be needed in order to confirm if RPE cells are visible with TOPI across the full 
accessible FOV for every subject. Importantly, the quality of RPE images was not impaired by individual skin phototypes and subsequent RPE pigmentation. TOPI examination also allowed accurate visualization of nerve fibre and vascular layers. Compared to images of retinal capillaries obtained with OCT angiography, the images of the smallest vasculature obtained with our method are independent of flow velocity and could thus provide complementary information.

The imaging modality can be easily adapted to a microscope and produce a powerful method to observe flat-mounted retina at the cellular level, without staining. Quantitative phase capability is obtained by combining multiple oblique illumination points and with appropriate modelling of the illumination function. For example, it allows the exploration of retinal capillaries and their surrounding cells.

The ability to image RPE cells within seconds in patients is of major interest to better understand the physiopathogenesis of retinal diseases and to follow the course of diseases that affect the RPE such as age-related macular degeneration (AMD), retinitis pigmentosa $^{30,31}$, or diabetic retinopathy ${ }^{32}$. Decrease in cell density and change in cell morphology could potentially be detectable at the early stage of AMD, before any complication has occurred ${ }^{33,34}$. TOPI could also be used to detect effects of drugs and treatments at a cellular level, offering the opportunity to intervene before irreversible functional loss has occurred. Morphologic quantitative new endpoints could thus emerge. In addition, recent medical studies have shown that Parkinson's and Alzheimer's diseases have an effect on the neuronal layers of the retina ${ }^{35,36}$, which may increase the impact of the clinical use of this technology.

In summary, TOPI holds promise for clinical use because it is, to the best of our knowledge, the only technique fast enough to image the RPE with high contrast and cellular resolution. Further clinical studies will clarify the limitations of this new imaging modality particularly in diseased states that distort the retinal layers organization. TOPI could not only accelerate our understanding of retinal diseases and define new morphological endpoints for therapeutic evaluation, but also the understanding of other neuro degenerative diseases.

\section{Materials and Methods}

\section{Study on human subjects}

The single-centre study aimed at validating the performances of our phase imaging device adheres to the tenets of the Declaration of Helsinki. The protocol involving healthy human volunteer subjects was approved by the local Ethics Committee of the Swiss Department of Health on research involving human subjects (CER-VD N²017-00976). Informed consent was obtained from the subjects. The left eyes of eleven healthy volunteers were imaged (four women, seven men) with an average age of 28 years (+/- 9) and having different skin pigmentations ranging from 1 to 6 on the Fitzpatrick scale were imaged ${ }^{26}$.

\section{In vivo experimental design}

The transscleral oblique illumination of the retina was produced using two Light Emitting Diodes (LED) with $810 \mathrm{~nm}$ nominal wavelength, $25 \mathrm{~nm}$ FWHM. The LED light beams are 
focused on the sclera at the pars-plana region to the temporal and nasal side of the iris (Fig. 2). The light beams can be operated at the same time or alternatively to capture images with one or two illumination points. The obtained angle of illumination for each point was therefore approximately $35^{\circ}$. The subject's head and eye were aligned with the system using an adjustable chin and forehead rest.

The apparatus optical diagram is shown in (Fig. 2) and consisted in the following elements: The illumination arm was composed of a yellow fixation target, of a 756-nm superluminescent diode (SLD, Exalos), which provided a point source at the retinal plane for the aberration correction measurements, and of an $1050 \mathrm{~nm}$ low power infrared LED for illuminating the pupil uniformly. The imaging arm integrated a pupil camera (DCC3240M, Thorlabs), an AO feedback loop ${ }^{10}$ (Imagine Eyes Mirao 52e or Alpao DM97-15 deformable mirror and Imagine Eyes HASO4 wavefront sensor), and an sCMOS camera for imaging the retina (ORCA Flash 4.0, Hamamatsu). The retinal sCMOS camera collected the light coming from the transscleral illumination to image the eye fundus through the pupil with a lateral sampling of $0.7 \mu \mathrm{m} /$ pixel.

TOPI images were acquired in healthy volunteers, in dark room conditions without pupil dilation. The pupil diameter ranged from 4 to $7 \mathrm{~mm}$. Once the position of the eye was aligned on the setup thanks to a chin and forehead rest, the retinal camera, which was mounted on a translation stage, was adjusted to focus on the targeted retinal layer. The realtime AO feedback loop was used for correcting the defocus accommodation of the eye (i.e., axial shifts of the plane), thus always locking a given imaging depth in the retina to a corresponding camera position. The depth sectioning is limited in this case by the depth of field, which is given by the pupil size and the aberrations of the eye. For instance, for an aberration-free-6-mm pupil, the depth of field is $27 \mu \mathrm{m}$, yielding a value of $0.17 \mathrm{NA}$ (the equivalent focal distance of the eye lens in air is $17 \mathrm{~mm}$ ), for a lateral resolution of $2.9 \mu \mathrm{m}$. A sequence of one or two hundred images at a given depth in the retina (100 with simultaneous points and 200 with alternate) was acquired by successively turning the LED spots $\mathrm{ON}$ and OFF with an exposure time of $8 \mathrm{~ms}$ and a repetition rate of $11 \mathrm{~Hz}$ per illumination point. The image focus can be made at any depth in the retina by introducing a quadratic phase shift (defocus term) on the deformable mirror. The maximum reflection point of the SLD probing beam is known to be at the photoreceptor layer. The defocus term is varied to reach a specific layer (such as RPE) and can be calibrated in advance by knowing the average distance between the photoreceptors and the layer of interest. The large depth of field (i.e. 27-100 $\mu$ m, depending on pupil size) of our non-confocal technique allow to image the RPE even if the real distance is slightly different from the calibrated one. Each layer took 9 seconds to image. The raw images were then post-processed, as explained in the "in vivo image processing" section, to produce a single TOPI image.

\section{Light safety analysis}

The illumination beams are projected on the participant's sclera when the eye is correctly aligned with the instrument. However, a complete safety analysis has to take into account any accidental projection of the beam on the skin, iris, cornea and lens to ensure no potential hazard of the system. To this end, the scleral, corneal, lens and retinal exposure were 
measured or calculated and compared with their respective exposure limits. We used the limits as expressed in the international standards for ophthalmic instrument optical safety ISO 15004-2:2007 for the ocular tissue and the skin limits given in the 2013 ICNIRP Guidelines on limits of exposure to incoherent visible and infrared radiation.

Eye exposure conditions-The beam minimum diameter is $3.5 \mathrm{~mm}$ at its focus point on the eye and the full converging angle is 0.37 radian. The pulse length is $8 \mathrm{~ms}$ and the repetition rate $11 \mathrm{~Hz}$ (duty cycle of 0.088 ), with a measured pulse power of $250 \mathrm{~mW}$ at a centre wavelength of $810 \mathrm{~nm}$ with FWHM of $25 \mathrm{~nm}$. The consecutive acquisition time is no longer than 10 seconds. Based on the measured power and pulse specification, the eye exposure is straightforward calculated:

Maximum pulsed irradiance at the sclera surface $(8 \mathrm{~ms}): \mathrm{E}_{\mathrm{P}}=2.598 \mathrm{~W} / \mathrm{cm}^{2}$.

Scleral radiant exposure for single pulse (1 pulse, $8 \mathrm{~ms}$ ): $\mathrm{H}_{1}=21 \mathrm{~mJ} / \mathrm{cm}^{2}$

Time-average irradiance $(11 \mathrm{~Hz}): \mathrm{E}_{\mathrm{AV}}=229 \mathrm{~mW} / \mathrm{cm}^{2}$.

Scleral radiant exposure for maximum exposure time (110 pulses, $10 \mathrm{~s}): \mathrm{H}_{110}=2.287 \mathrm{~J} / \mathrm{cm}^{2}$

Retinal exposure-The light exposure reaching the underlying choroid and retina after crossing the sclera is less straightforward to compute. Only part of the light will transmit, and the beam will be scattered and spread by the tissue. The more accurate measurement on scleral transmission and absorption were done by Vogel et al. in $1991^{20}$. They measured a $\sim 33 \%$ transmission (and $\sim 5 \%$ absorption) for a wavelength of $810 \mathrm{~nm}$. As a conservative estimate, we will assume no diffusion nor beam spreading, thus giving a retinal exposure of $33 \%$ of the scleral exposure to compare with the limits.

The retinal irradiance in case of accidental trans-pupil illumination is calculated thanks to the beam converging angle. The retinal image diameter $d_{r}$ and surface $A$ is:

$$
\mathrm{d}_{\mathrm{r}}=\mathrm{f} \cdot \varphi=(17 \mathrm{~mm})(0.37 \text { radian })=6.29 \mathrm{~mm} \rightarrow \mathrm{A}=0.31 \mathrm{~cm}^{2}
$$

The irradiance is then directly found: $\mathrm{E}=250 \mathrm{~mW} / 0.31 \mathrm{~cm}^{2}=0.805 \mathrm{~W} / \mathrm{cm}^{2}$. The irradiance of the posterior retina in case of accidental trans-pupil exposure $\left(0.805 \mathrm{~W} / \mathrm{cm}^{2}\right)$ is smaller than the irradiance of the retina underlying the sclera in case of intended transscleral exposure $\left(33 \% * 2.598=0.857 \mathrm{~W} / \mathrm{cm}^{2}\right)$. The transscleral exposure and not the accidental trans-pupil exposure is therefore the worst-case exposure scenario for the retina to compare with the limits.

Applicable limits for Group 1 pulsed instrument-Weighted retinal visible and infrared radiation radiant exposure limit:

$$
\text { For } 1 \text { pulse }(8 \mathrm{~ms}): \mathrm{H}_{\text {VIR-R }}=0.1605 \mathrm{~J} / \mathrm{cm}^{2}
$$


For 110 pulses(10s): HVIR-R $=10.4184 \mathrm{~J} / \mathrm{cm}^{2}$

Unweighted corneal and lenticular infrared radiation radiant exposure limit:

$$
\text { For } 1 \text { pulse( } 8 \mathrm{~ms}): \mathrm{H}_{\mathrm{IR}-\mathrm{CL}}=0.5383 \mathrm{~J} / \mathrm{cm}^{2}
$$

For 110 pulses $(10 \mathrm{~s}): \mathrm{H}_{\mathrm{IR}-\mathrm{CL}}=3.2009 \mathrm{~J} / \mathrm{cm}^{2}$

Unweighted anterior segment visible and infrared radiation radiant exposure limit (for convergent beam only):

$$
\text { For } 1 \text { pulse(8ms):HVIR-AS }=7.4767 \mathrm{~J} / \mathrm{cm}^{2}
$$

For 110 pulses(10s): $\mathrm{H}_{\text {VIR-AS }}=44.4570 \mathrm{~J} / \mathrm{cm}^{2}$

\section{Skin limits-}

$$
\text { For } 1 \text { pulse }(8 \mathrm{~ms}): \mathrm{H}_{\mathrm{SKIN}}=0.5981 \mathrm{~J} / \mathrm{cm}^{2}
$$

$$
\text { For } 110 \text { pulses(10s): } \mathrm{H}_{\mathrm{SKIN}}=3.5566 \mathrm{~J} / \mathrm{cm}^{2}
$$

The radiant exposure conditions of the eye for 1 pulse $H_{1}$ and 110 pulses $H_{110}$ calculated above can be directly compared with the different limits. They are below all limits for Group 1 instrument (i.e. "ophthalmic instrument for which no potential light hazard exists") as well as below the ICNIRP skin limits. The retina limits for our NIR exposure are not even attained if the sclera were considered with $100 \%$ transmission. The safety factor for retinal exposure is therefore at least a factor 20 (considering 33\% transmission and thermal hazard weighting function $R(\lambda)=0.6$ for $\lambda=810 \mathrm{~nm})$.

The condition being the closest to the limit is the cornea and lenticular exposure in case of misalignment of the device, or a subject starring directly at the illumination beam. This event is very unlikely to happen but a safety factor of 1.5 prevent any potential adverse effect in that case.

No explicit limits are provided for the sclera in the ISO 15004-2:2007 or ICNIRP guidelines. However, limits are provided for the anterior segment (cornea and lens) and for the skin. The sclera tissue itself can be considered in between skin and cornea. As it has a similar absorption coefficient for NIR as the cornea (but more scattering) ${ }^{20}$, the sclera is expected to have a similar exposure limit. It is therefore reasonable to assume that sclera is safe if both 
the anterior segment limits and the limits for the more pigmented tissues such as skin are not attained, which it is the case for our instrument. As an additional safety evidence, far larger radiant exposure was sent on the sclera by Geffen et al. without noticing any adverse effect $^{37}$.

\section{In vivo image processing}

Because of sudden eye micro-saccades during the $8 \mathrm{~ms}$ acquisition time, some raw images had motion-blur artefacts. Therefore, only the $80 \%$ sharpest images were automatically or manually selected for each illumination point based on the contrast criteria ( $\mathrm{std} / \mathrm{mean}$ ). The images were then aligned in order to correct for the eye motions using the ImageJ plugin TurboReg, which considers the translations and the rotations of the images ${ }^{38}$. Once the raw images were aligned, averaging of the sequence produced a single high-SNR dark-field image for each illumination point for a specific layer. The two dark-field images were then summed to produce the final image. When the two illumination points are emitting together, only one dark field image of equivalent contrast is obtained. For visualization, the dark-field image was high-pass filtered (ImageJ bandpass filter with boundaries 1pixel-30pixels) and normalized with histogram stretching. The high-pass filter was used to remove a large part of the out-of-focus light.

\section{Cell segmentation and quantification}

Cell segmentation was performed on several retinal layers to obtain quantitative morphology and density values. For the RPE layer, an automatic segmentation was performed via local minimum detection. Then, the Voronoi filter function was applied to create equidistant cell contours between the two nearest maxima, leading to RPE segmentation. Voronoi mapping is commonly used to map the retinal soma mosaic ${ }^{9}$. The coefficient of variation $(\mathrm{CoV})^{39}$, has been computed on subject S10 based on automatic RPE count and area measurements repeated five times under changed equipment and materials (two different deformable mirrors and two different head mounts were used) over five months. The results, plotted on Supplementary Fig. 10, give a CoV of 6.13\% for RPE count and $6.44 \%$ for RPE area. This standard measurement characterizes the reproducibility of the acquisition. The resulting values are considered good for a prototype device since higher values of $\mathrm{CoV}$ can be measured on commercial devices ${ }^{39}$.

\section{Statistical analysis}

Results were expressed as mean \pm standard deviation. Student's t-test was used to compare our data with that of the literature, when possible. A $P$ value of $<0.05$ was considered statistically significant.

\section{Retinal ex-vivo samples}

Flat-mounted retina-choroid complexes-The two human-donor eyes were obtained from the eye bank of the Jules-Gonin Eye Hospital, in conformity with the Swiss Federal law on transplantation. It was collected for research purpose at $10 \mathrm{~h}$ post-mortem, fixed in a $4 \%$ paraformaldehyde solution (PFA) for $24 \mathrm{~h}$, and then stored in $1 \% \mathrm{PFA}$ at $+4^{\circ} \mathrm{C}$ until it was analysed. Pig eyes were obtained from a local slaughterhouse, a few hours after the 
animals' death. These eyes were fixed in 4\% PFA for $24 \mathrm{~h}$, and then stored in a phosphatebuffered saline (PBS) at $+4^{\circ} \mathrm{C}$ for one day before mounting.

After washing in PBS, the anterior segment of the eyes and the vitreous were removed. The retina-choroid complexes were detached from the remaining posterior segments, and flatmounted in a solution made of PBS-glycerol (1:1). The human retina-choroid complexes were observed using a confocal microscope (Zeiss LSM710). The human and pig samples were also observed using our experimental phase microscope (as illustrated in Supplementary Fig. 6D).

Cryosectioning-One pig eye was kept for cryosectioning. After fixation and microdissection, the posterior segment of the eye was snap- frozen prior to frozen sectioning on a microtome-cryostat. Frontal 10 um-thick sections were prepared and mounted on a glass coverslip. These sections were imaged using a digital holographic microscope $(\mathrm{DHM})^{40}$ and then with our phase microscope.

Immunohistochemistry on neuroretinas-Two pigmented rats from the animal facility of the Jules-Gonin Eye Hospital were used. Investigations were performed in accordance of the Association for Research in Vision and Ophthalmology (ARVO) statement for the Use of Animals in Ophthalmic Vision Research. Our study was approved by the cantonal veterinary office (Authorization VD2928). Retinal morphology was examined invivo, under ketamine / xylazine $(80 \mathrm{mg} / \mathrm{kg}$ and $8 \mathrm{mg} / \mathrm{kg}$ ) anaesthesia and after dilation, using a spectral domain OCT system adapted for rat eyes (Bioptigen). Animals were sacrificed via pentobarbital injection. After enucleation and fixation of the whole eyes during $2 \mathrm{~h}$ in $4 \%$ PFA, the anterior segments were discarded and the neuroretinas were carefully separated from the remaining posterior segment. Post-fixation was carried out for $10 \mathrm{~min}$ at $-20^{\circ} \mathrm{C}$ in acetone.

For pericytes immunostaining, after rehydration in PBS supplemented with $0.5 \%$ Triton $\mathrm{X} 100$ and $10 \%$ foetal bovine serum (FBS) overnight at $+4^{\circ} \mathrm{C}$, the retinas were incubated for two days at $+4^{\circ} \mathrm{C}$ with polyclonal rabbit anti-NG2 antibodies (1:200; AB5320, Merk Millipore). For microglia immunostaining, after rehydration in PBS supplemented with $0.5 \%$ Triton X100 and 10\% FBS for 30 minutes at room temperature, the retinas were incubated overnight at $+4{ }^{\circ} \mathrm{C}$ with a rabbit anti-iba1 (1:200; Wako) and a goat anti-collagen IV (1:100, Serotec) antibodies diluted in the same buffer. After washing, the neuroretinas were incubated with the appropriate antibody (Alexa-488-conjugated goat anti rabbit IgG, 1:250; donkey anti-goat A633, 1:400; Invitrogen), diluted in PBS supplemented with 0.1\% Triton X100 and 10\% FBS, for $2 \mathrm{~h}$ at room temperature. After washing, the tissues were stained for 10 min with 4', 6-Diamidino-2-Phenyl-Indole (DAPI, 1:10 000), washed again, and flat-mounted in PBS-glycerol (1:1). The neuroretinas were examined with a confocal microscope (Zeiss LSM 710), and then with our phase microscope.

\section{Ex-vivo experimental design}

Our experimental phase microscope is illustrated in Supplementary Fig. 6. A total of four different LEDs were placed equally spaced around a microscope objective to produce four different illumination points. The sample was placed below the microscope objective (MO) 
and imaged using a CMOS camera using the MO and an achromatic doublet as a tube lens. A scattering layer of translucent commercial tape was used between each LED and the sample to increase the extent of illumination light and to mimic the scattering caused by the sclera. The sample was moved vertically to change the imaging layer depth. Depending on the NA of the MO, the exposure time varied between $1 \mathrm{~ms}$ and $20 \mathrm{~ms}$. For each illumination point, a total of ten raw images were averaged to increase the SNR. A total of four averaged dark-field images (one for each points) were obtained for each depth. Then, the processing steps described below were carried out to produce either differential phase contrast (DPC) images or a single phase-contrast image.

\section{Ex-vivo image processing and phase reconstruction}

An image of the sample is recorded for each illumination point and a final quantitative phase image is reconstructed following the method described in a previous paper ${ }^{24}$. Phase images can be reconstructed using the following principle: at least two pictures with intensities I(a) and $\mathrm{I}(a+180)$, captured with two different symmetric illumination polar angles ( $a$ and $a$ $+180^{\circ}$ ), are required. A DPC image can be then obtained according to:

$$
I_{D P C}=\frac{I(\alpha)-I\left(\alpha+180^{\circ}\right)}{I(\alpha)+I\left(\alpha+180^{\circ}\right)} .
$$

Using several illumination points, multiple DPC images are produced. By combining these different DPC images of the same object, it is possible to obtain a quantitative phase image of the object thanks to the reconstruction process described in Tian and Waller' study ${ }^{41}$. Finally, the reconstruction process of the phase is performed ${ }^{24,41,42}$. The reconstruction process is a Tikhonov regularization process with a known transfer function. The absorption transfer function is supposed to be identical for the different illumination points, meaning that it cancels out after the subtraction. The phase transfer function was calculated using the linearized imaging model presented by Tian and Waller ${ }^{41}$.

\section{Supplementary Material}

Refer to Web version on PubMed Central for supplementary material.

\section{Acknowledgments}

The authors thank Michaël Nicolas from the eye bank of the Jules-Gonin Eye Hospital for providing a post-mortem human eye, Irmela Mantel together with the team of the centre for clinical investigation for their time spent in performing the ophthalmologic checks for our study on healthy participants, and Sylvain Roy, Alexandre Matet and Daniel Sage for the fruitful discussions.

Funding

In addition to the research partners, this study was supported by the following programs: Enable program of the Technology Transfer Office at EPFL (610263), EPFL Innogrant (INNO 17-15), Bridge proof of concept (InnoSuisse and SNSF, 20B1-1_178253), Gebert Rüf Stiftung foundation (GRS-052/17) and EIT Health Innovation by idea (19323-ASSESS). 


\section{References}

1. Wong W, et al. Global prevalence of age-related macular degeneration and disease burden projection for 2020 and 2040: a systematic review and meta-analysis. The Lancet Glob Health. 2014; 2(2):106-116.

2. Pallikaris A, Williams DR, Hofer H. The Reflectance of Single Cones in the Living Human Eye. Invest Ophthalmol Vis Sci. 2003; 44(10):4580-92. [PubMed: 14507907]

3. Gao W, Cense B, Zhang Y, Jonnal RS, Miller DT. Measuring retinal contributions to the optical Stiles-Crawford effect with optical coherence tomography. Opt Express. 2008; 16(9):6486-6501. [PubMed: 18516251]

4. Artal P, Navarro R. High-resolution imaging of the living human fovea: measurement of the intercenter cone distance by speckle interferometry. Opt Lett. 1989; 14(20):1098-1100. [PubMed: 19753067]

5. Fernández EJ, Iglesias I, Artal P. Closed-loop adaptive optics in the human eye. Opt Lett. 2001; 26(10):746-748. [PubMed: 18040440]

6. Viard C, et al. Imaging microscopic structures in pathological retinas using a flood illumination adaptive optics retinal camera. Ophthalmic Technologies XXI. 2011

7. Gofas-Salas E. High loop rate adaptive optics flood illumination ophthalmoscope with structured illumination capability. Appl Opt. 2018; 57(20):5635-5642. [PubMed: 30118075]

8. Gofas-Salas E. Near infrared adaptive optics flood illumination retinal angiography. Biomed Opt Express. 2019; 10(6):2730-2743. [PubMed: 31259047]

9. Liu Z, Kurokawa K, Zhang F, Lee JJ, Miller DT. Imaging and quantifying ganglion cells and other transparent neurons in the living human retina. PNAS. 2017; 114(48):12803-12808. [PubMed: 29138314]

10. Miller DT, Kocaoglu OP, Wang Q, Lee S. Adaptive optics and the eye (super resolution OCT). Eye. 2011; 25(3):321-330. [PubMed: 21390066]

11. Rossi EA, et al. Imaging individual neurons in the retinal ganglion cell layer of the living eye. PNAS. 2017; 114(3):586-591. [PubMed: 28049835]

12. Guevara-Torres A, Williams DR, Schallek J. Imaging translucent cell bodies in the living mouse retina without contrast agents. Biomed Opt Express. 2015; 6(6):2106-2119. [PubMed: 26114032]

13. Chui TY, Gast TJ, Burns SA. Imaging of vascular wall fine structure in human retina using adaptive optics scanning laser ophthalmoscopy. Investig Ophthalmol Vis Sci. 2013; 54(10):71157124. [PubMed: 24071955]

14. Chui TYP, Van Nasdale DA, Burns SA. The use of forward scatter to improve retinal vascular imaging with an adaptive optics scanning laser ophthalmoscope. Biomed Opt Express. 2012; 3(10):2537-2549. [PubMed: 23082294]

15. Cunefare D, et al. Automated Detection of Cone Photoreceptors in Split Detector Adaptive Optics Scanning Light Ophthalmoscope Images. Investig Ophthalmol Vis Sci. 2016; 57(12):61-61.

16. Sulai YN, Scoles D, Harvey Z, Dubra A. Visualization of retinal vascular structure and perfusion with a nonconfocal adaptive optics scanning light ophthalmoscope. J Opt Soc Am A. 2014; 31(3):569-579.

17. Scoles D, Sulai YN, Dubra A. In vivo dark-field imaging of the retinal pigment epithelium cell mosaic. Biomed Opt Express. 2013; 4(9):1710-1723. [PubMed: 24049692]

18. Granger CE, et al. Human Retinal Pigment Epithelium: In Vivo Cell Morphometry, Multispectral Autofluorescence, and Relationship to Cone Mosaic. Investig Ophthalmol Vis Sci. 2018; 59(15):5705-5716. [PubMed: 30513531]

19. Liu Z, Kocaoglu OP, Miller DT. 3D Imaging of Retinal Pigment Epithelial Cells in the Living Human Retina. Investig Ophthalmol Vis Sci. 2016; 57(9):533-543. [PubMed: 26873512]

20. Vogel A, Dlugos C, Nuffer R, Birngruber R. Optical properties of human sclera, and their consequences for transscleral laser applications. Lasers Surg Med. 1991; 11(4):331-340. [PubMed: 1895865]

21. Geeraets $\mathrm{W}$, et al. The relative absorption of thermal energy in retina and choroid. Investig Ophthalmol Vis Sci. 1962; 3(1):340-347. 
22. Delori FC, Pflibsen KP. Spectral reflectance of the human ocular fundus. Appl Opt. 1989; 28(6):1061-1077. [PubMed: 20548621]

23. JGiese JD, Ford TN, Mertz J. Fast volumetric phase-gradient imaging in thick samples. Opt Express. 2014; 22(1):1152-1162. [PubMed: 24515075]

24. Carpentras D, Laforest T, Künzi M, Moser C. Effect of backscattering in phase contrast imaging of the retina. Opt Express. 2018; 26(6):6785-6795. [PubMed: 29609366]

25. Liang J, Williams DR, Miller DT. Supernormal vision and high-resolution retinal imaging through adaptive optics. J Opt Soc Am A. 1997; 14(11):2884-2892.

26. Fitzpatrick TB. The Validity and Practicality of Sun-Reactive Skin Types I Through VI. Arch Dermatol. 1988; 6(126):869-871.

27. Morgan JIW, Dubra A, Wolfe R, Merigan WH, Williams DR. In Vivo Autofluorescence Imaging of the Human and Macaque Retinal Pigment Epithelial Cell Mosaic. Investig Ophthalmol Vis Sci. 2009; 50(3):1350-1359. [PubMed: 18952914]

28. Bhatia SK, et al. Analysis of RPE morphometry in human eyes. Mol Vis. 2016; 22:898-916. [PubMed: 27555739]

29. Zhao M, et al. A new CRB1 rat mutation links Müller glial cells to retinal telangiectasia. J Neurosci. 2015; 35(15):6093-6106. [PubMed: 25878282]

30. Bhutto I, Lutty G. Understanding age-related macular degeneration (AMD): relationships between the photoreceptor/retinal pigment epithelium/Bruch's membrane/choriocapillaris complex. Mol Aspects Med. 2018; 33(4):295-317.

31. Humayun M. Morphometric analysis of the extramacular retina from postmortem eyes with retinitis pigmentosa. Invest Ophthalmol Vis Sci. 1999; 40(1):143-148. [PubMed: 9888437]

32. Rothschild P. ROCK-1 mediates diabetes-induced retinal pigment epithelial and endothelial cell blebbing: Contribution to diabetic retinopathy. Sci Rep. 2017; 7(1):8834. [PubMed: 28821742]

33. Fisher CR, Ferrington DA. Perspective on AMD Pathobiology: A Bioenergetic Crisis in the RPE. Invest Ophthalmol Vis Sci. 2018; 59(14):41-47.

34. Zhang Q, et al. Comparison of histologic findings in age-related macular degeneration with RPE flatmount images. Mol Vis. 2019; 25:70-78. [PubMed: 30820143]

35. London A, Benhar I, Schwartz M. The retina as a window to the brain-from eye research to CNS disorders. Nat Rev Neurol. 2013; 9(1):44-53. [PubMed: 23165340]

36. Morgia CL, Ross-Cisneros FN, Sadun AA, Carelli V. Retinal ganglion cells and circadian rhythms in Alzheimer's disease, Parkinson's disease, and beyond. Front Neurol. 2017; 8

37. Geffen N, et al. Transscleral selective laser trabeculoplasty without a gonioscopy lens. J Glaucoma. 2017; 26(3):201-207. [PubMed: 27636593]

38. Thévenaz P, Ruttimann UE, Unser M. A pyramid approach to subpixel registration based on intensity. IEEE Trans Image Process. 1998; 7(1):27-41. [PubMed: 18267377]

39. Strouthidis N, White NE, Owen V, Ho T, Hammond C, Garway-Heath D. Factors affecting the testretest variability of Heidelberg retina tomograph and Heidelberg retina tomograph II measurements. Br J Ophthalmol. 2005; 89(11):1427-1432. [PubMed: 16234446]

40. Monemhaghdoust Z, Montfort F, Emery Y, Depeursinge C, Moser C. Off-axis digital holographic camera for quantitative phase microscopy. Biomed Opt Express. 2014; 5(6):1721-1730. [PubMed: 24940535]

41. Tian L, Waller L. Quantitative differential phase contrast imaging in an LED array microscope. Opt Express. 2015; 23(9):11394-11403. [PubMed: 25969234]

42. Mehta SB, Sheppard CJR. Quantitative phase-gradient imaging at high resolution with asymmetric illumination-based differential phase contrast. Opt Letters. 2009; 34(13):1924-1926. 

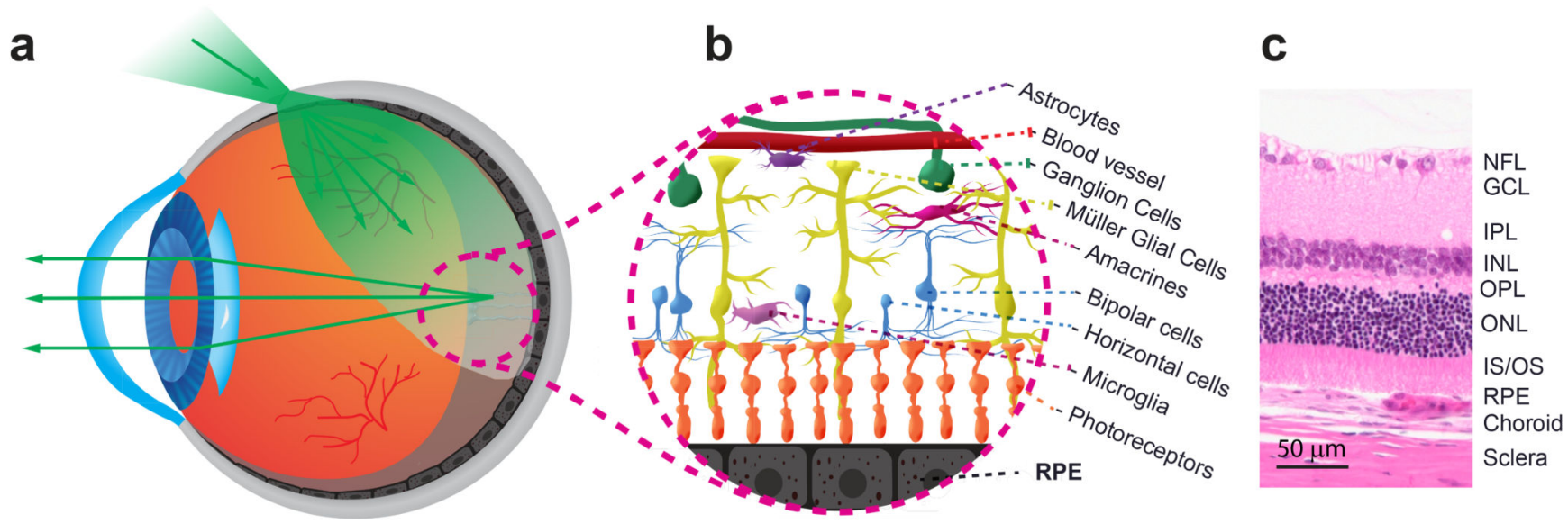

Fig. 1. Illumination of the retinal layers provided via transscleral illumination.

(a) Light transmission into the eye. The light is first transmitted through the sclera, the retina pigment epithelium (RPE), and the neuroretina. After travelling through the vitreous humor and the retina, it impinges on the RPE layer. The light is multiply scattered by the RPE. A part travels back and propagates through the neuroretina and its translucent cells, the vitreous, the eye lens, and, then, the anterior segment of the eye. (b) Artistic illustration of the translucent vascular, neuronal and glial cells of the retina. (c) Histological cut of the retinal layers, the choroid, and the sclera. GCL: ganglion cells layer; INL: inner nuclear layer; IPL: inner plexiform layer; IS/OS: inner and outer segments of the photoreceptors; NFL: nerve fiber layer; ONL: outer nuclear layer; OPL: outer plexiform layer. 


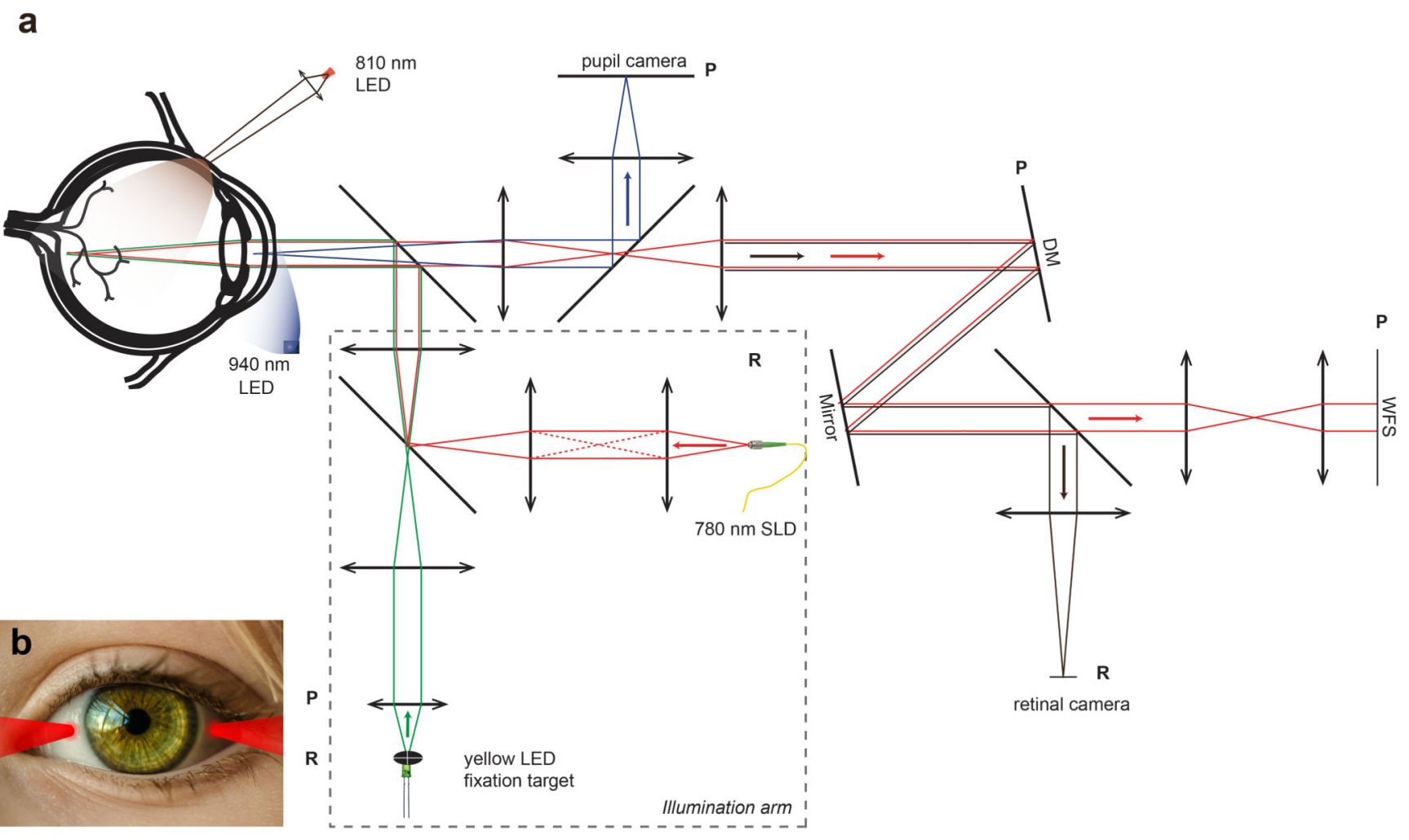

Fig. 2. Optical diagram of the in vivo Transcleral Optical Phase Imaging system.

a) Transscleral light is produced by two 810nm LEDs focused on the sclera surface (one is shown). Light is then transmitted inside the eyeball. After scattering from the retinal cell layers, the light going out of the eye pupil is collected by the optical system. Other light sources are 940nm LED for pupil retro illumination, $780 \mathrm{~nm}$ SLD for aberration measurement and yellow 580nm LED for fixation target. The optical setup includes a pupil camera for alignment, a retinal camera to record fundus image and an adaptive optics loop to correct the aberration of the eye. DM: Deformable mirror; LED: light-emitting diode; SLD: superluminescent diode; WFS: wavefront sensor. P planes are eye pupil conjugated planes. $\mathrm{R}$ planes are retina conjugated planes. b) Illustration of transscleral illumination by the two $810 \mathrm{~nm}$ LED focused beams on the sclera. 

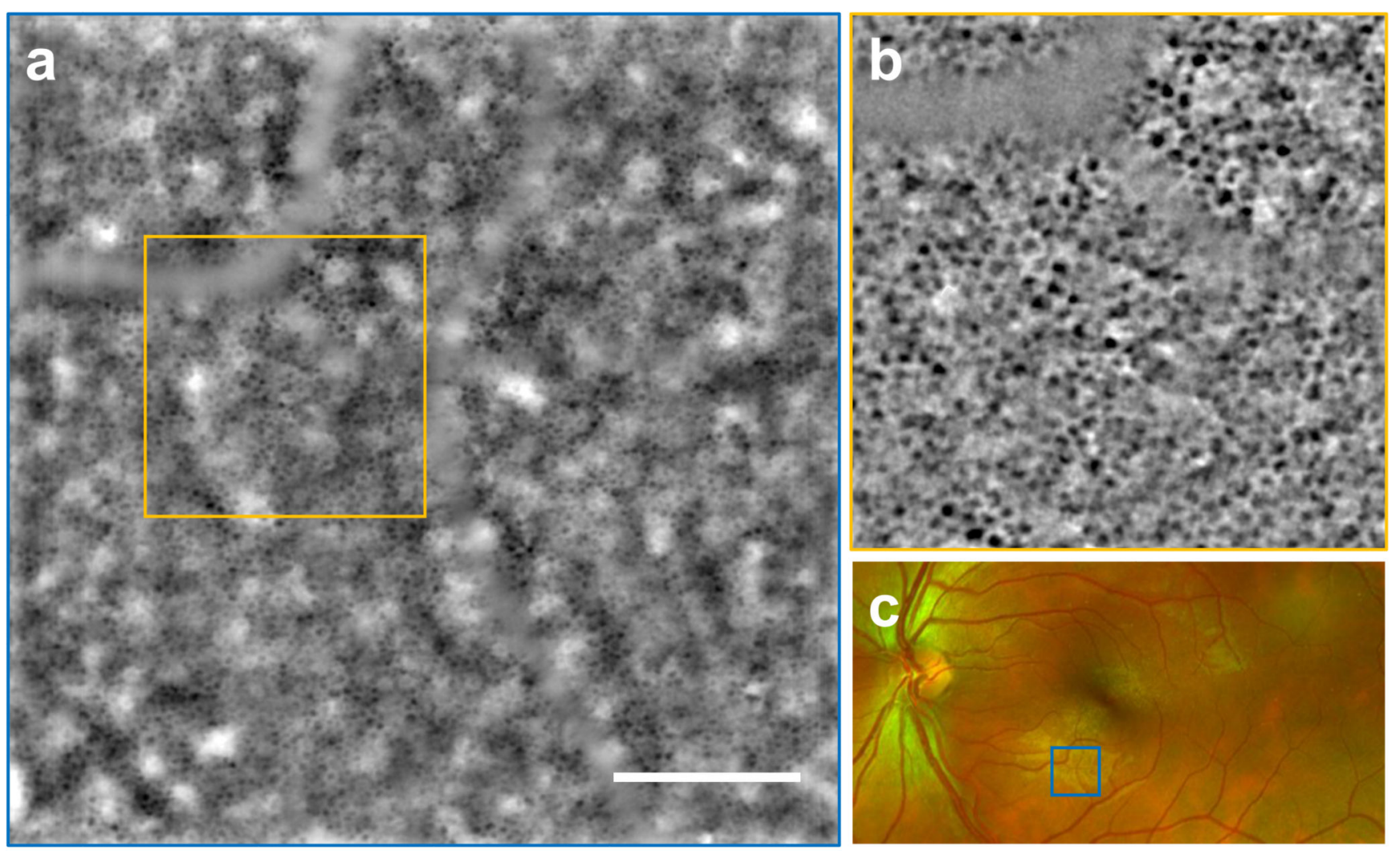

Fig. 3. In vivo full-field $\left(4.4^{\circ} \times 4.4^{\circ}\right)$ image of the retinal pigment epithelium (RPE) of a healthy volunteer (left eye, subject S10) taken with TOPI at an eccentricity of $6.7^{\circ}$ from the fovea.

(a) The RPE image is the average of 100 raw retinal images taken in 9 seconds to increase the SNR. A mosaic of RPE cells is visible where the individual cell somas appear dark due to the absorbing pigment within the cell body. The cells are partially masked under a lower spatial frequency background modulation with varying intensity, coming from the out-offocus signal from the choroid. Scale bar, $1^{\circ}$ or $300 \mu \mathrm{m}$. Orange square shows the inset "b" location. (b) The original RPE image is cropped and high-pass filtered to remove the out of focus light and have a more uniform cell mosaic (c) SLO fundus image showing the location of the TOPI image "a" (blue square). 

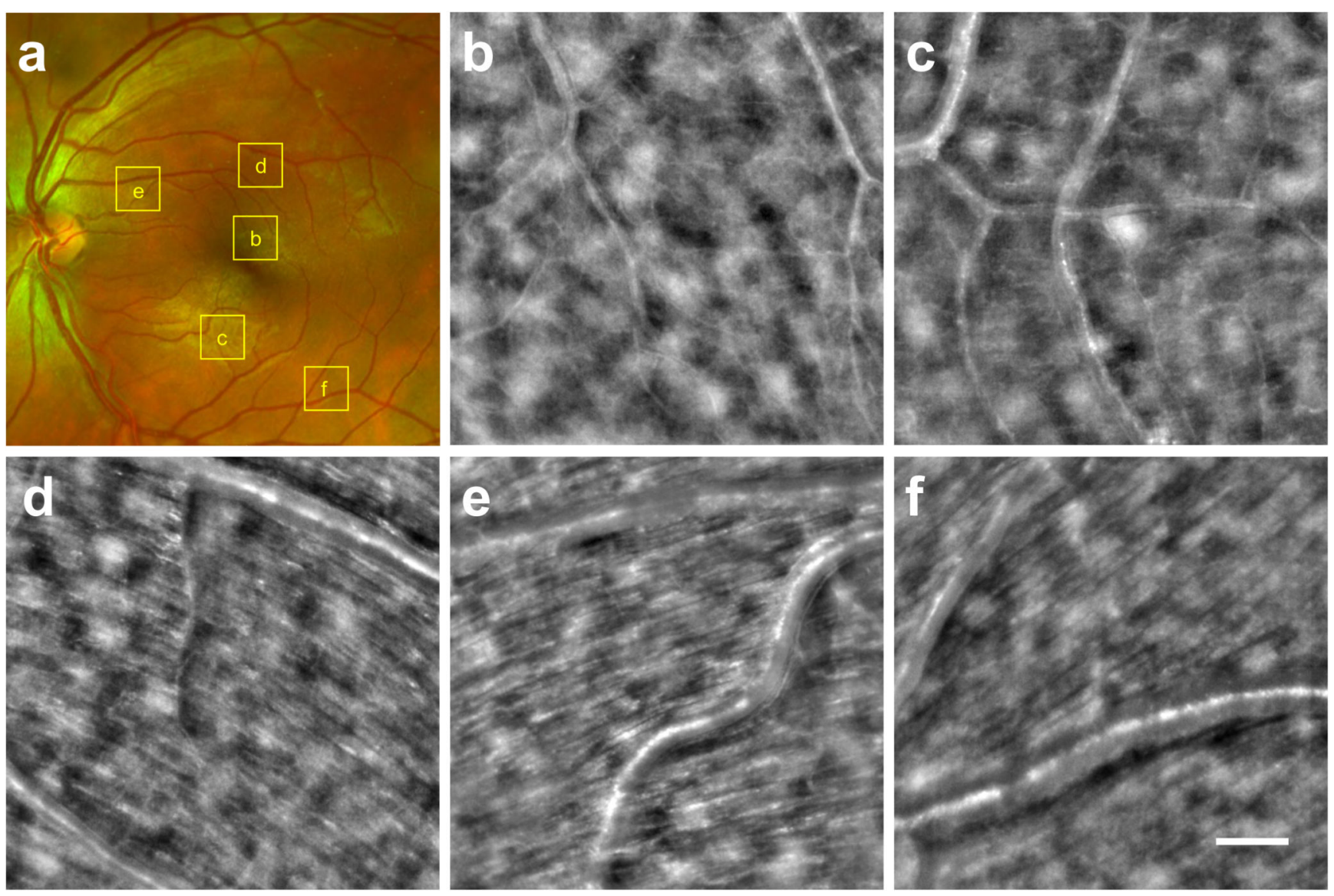

Fig. 4. Microcapillaries and nerve fiber layer TOPI images of subject S10 taken at different eccentricities.

Each $3^{\circ} \times 3^{\circ}$ field of view image is cropped from a full TOPI image for better visualization in the figure. Scale bar, $0.5^{\circ}$ or $150 \mu \mathrm{m}$. (a) SLO fundus image showing the location of each TOPI image crop. (b-c) Microcapillaries being few microns thick are visible even if no blood flow is present thanks to the dark field effect of the oblique illumination. (d-e-f) Large axons bundles of 30-50 $\mu \mathrm{m}$ are clearly identifiable when looking at the nerve fiber layer. The bundles are well aligned and directed toward the optic nerve, as expected. 


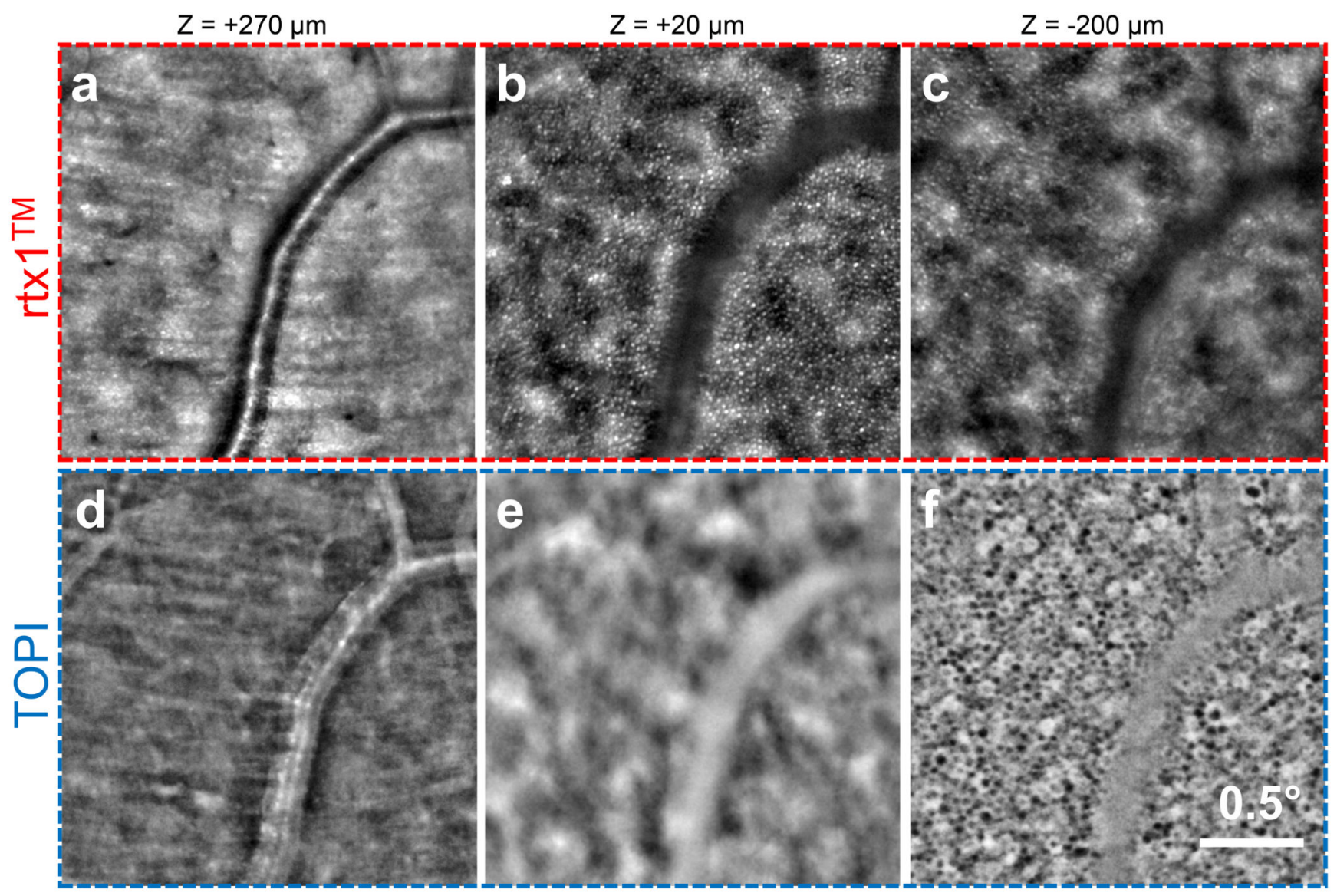

Fig. 5. Comparison between in vivo images from a trans-pupil flood illumination commercial system (rtx1 ${ }^{\mathrm{TM}}$, Imagine Eyes; Top) and a trans-scleral illumination TOPI image (Bottom) on the same area.

(a, b) In the inner retina $\left(\mathrm{z}_{\mathrm{rtx} 1}=270 \mu \mathrm{m}\right)$, the blood vessel appears light on dark background with TOPI due to the darkfield effect of oblique illumination. (b, e) At the photoreceptor level $\left(\mathrm{z}_{\mathrm{rtx} 1}=20 \mu \mathrm{m}\right)$, the cones appear hyper-reflective in the $\mathrm{rtx} 1$ image due to light coupling inside the cells (Stiles-Crawford effect). They are barely visible in the TOPI image because no reflection is produced by the oblique illumination. (c, f) At the RPE level $\left(\mathrm{z}_{\mathrm{rtx} 1}=-200\right.$ $\mu \mathrm{m})$, on the rtx1 image, no RPE cells are visible because their signal is buried under the outof-focus photoreceptors light, whereas on the TOPI image, the RPE cell mosaic is visible. The somas appear dark because of the pigments composing the cells. 

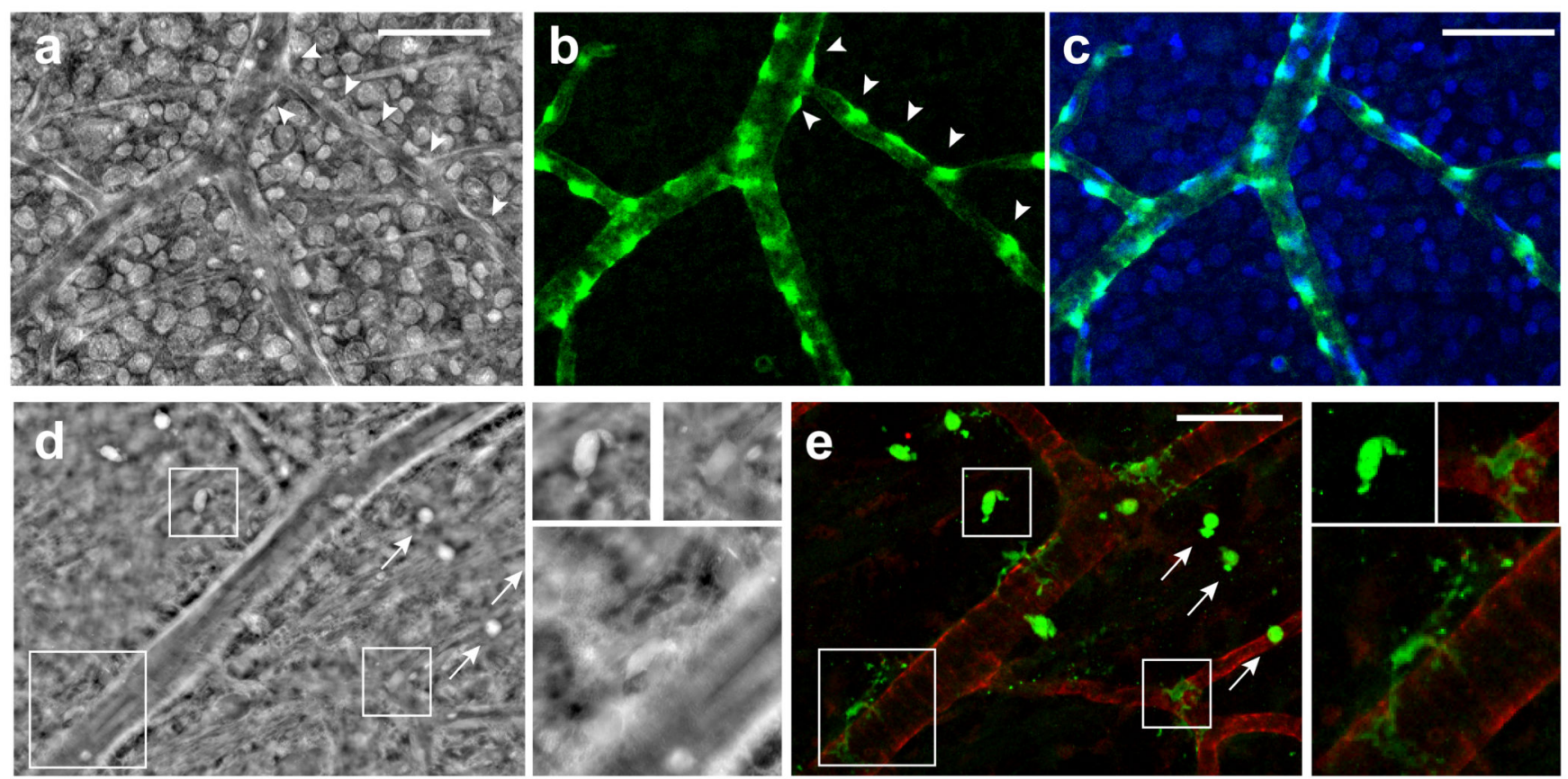

Fig. 6. Image correlation analysis between oblique illumination optical phase imaging (OPI) microscopy and fluorescence confocal microscopy of retinal vessels and their surrounding cells. (a-c) Pericytes visualization in a healthy retina from a 3-month-old rat. Cells highlighted with arrowheads in the phase image obtained under a $0.4-\mathrm{NA}$ objective (a) were localized exactly where the NG2+ pericytes were detected in fluorescence images (in green, b-c). Nuclei are stained in blue (c). (d-e) Microglial cells visualization in a retina presenting retinal dystrophy from an 11-month-old rat. Phase images obtained under a 0.65-NA objective (d) were compared with fluorescence images (e) showing Iba1-positive microglia (in green) and collagen IV-positive vessels (in red). Magnifications of three cells in both imaging modalities illustrate the detection of microglial cells using the OPI modality (d). Ramified quiescent microglia and reactive microglia, with a round cell body (arrows), are shown. Scales bar, $50 \mu \mathrm{m}$. 